\title{
Alcohol-Responsive Writer's Cramp
}

\author{
Sung-Chul Lim ${ }^{1}$, Joong-Seok Kim ${ }^{1}$, Jae-Young $\mathrm{An}^{1}$ and Sa Yoon Kang ${ }^{2}$
}

\begin{abstract}
Writer's cramp is a rare movement disorder of unknown etiology, in which a cramp is elicited primarily or exclusively with writing. We describe a patient with primary writer's cramp that was completely improved by drinking a small amount of alcohol. Although it is unclear how "alcohol" ameliorated the dystonia, this case suggests that alcohol might reverse the pathophysiologic changes in the entire basal ganglia circuit. In addition, we cannot rule out the possibility that the anxiolytic influence of alcohol may contribute to the beneficial effects on dystonia.
\end{abstract}

Key words: writer's cramp, alcohol

(Intern Med 51: 99-101, 2012)

(DOI: 10.2169/internalmedicine.51.6322)

\section{Introduction}

Writer's cramp is the most common task-specific action dystonia, elicited primarily or exclusively with writing. In most cases, this disorder is associated with discomfort, frequently includes other tasks, and often produces significant disability with no identifiable drug responses (1). In this report, we present a 43-year-old woman with a 20-year history of writer's cramp. The use of a small amount of alcohol produced transient and complete relief of symptoms.

\section{Case Report}

A 43-year-old right-handed female who was a retired public servant, was referred to our movement clinic for evaluation of a 20-year history of right hand dystonia during writing. At 23 years of age, she suffered the sudden onset of dystonic sense during a written examination. Since that time, her right hand developed an abnormal posture whenever she wrote. However, she did not have any discomfort or pain while engaged in other activities. The dystonia was not present until she grasped a writing instrument. The symptom was not affected by sleep, however, emotional stress made the symptoms worse. The patient had been treated with benzodiazepine, trihexiphenidyl and baclofen, although there were no benefits to these drugs. However, the patient reported her symptoms were temporally relieved by drinking a small amount of alcohol. The patient was never exposed to neuroleptics nor did she have a family history of dystonia.

There was no dystonia or myoclonus of the limbs, with routine movement. Examination of the patient during handwriting showed a combination of excessive squeezing of the pen with the thumb and index finger, and involuntary extension of the wrist. Writing was slow and effortful, but not associated with pain (Fig. 1A). Other sensory stimuli, such as touch and pressure, did not improve the writing movement.

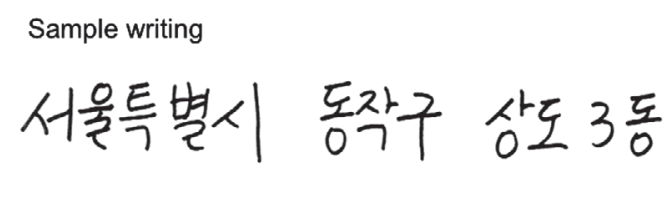

A

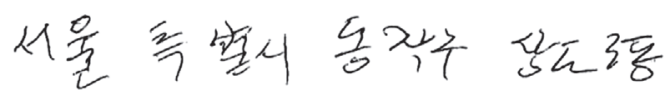

B

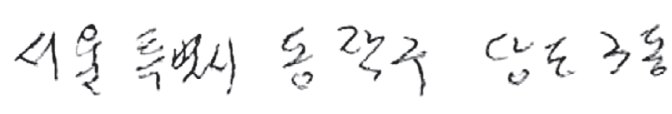

Figure 1. A note written by the patient prior to drinking a small amount of alcohol (A) and after drinking alcohol (B).

${ }^{1}$ Department of Neurology, College of Medicine, The Catholic University of Korea, South Korea and ${ }^{2}$ Department of Neurology, College of Medicine, Cheju National University, South Korea

Received for publication August 3, 2011; Accepted for publication September 16, 2011

Correspondence to Dr. Joong-Seok Kim, neuronet@catholic.ac.kr 


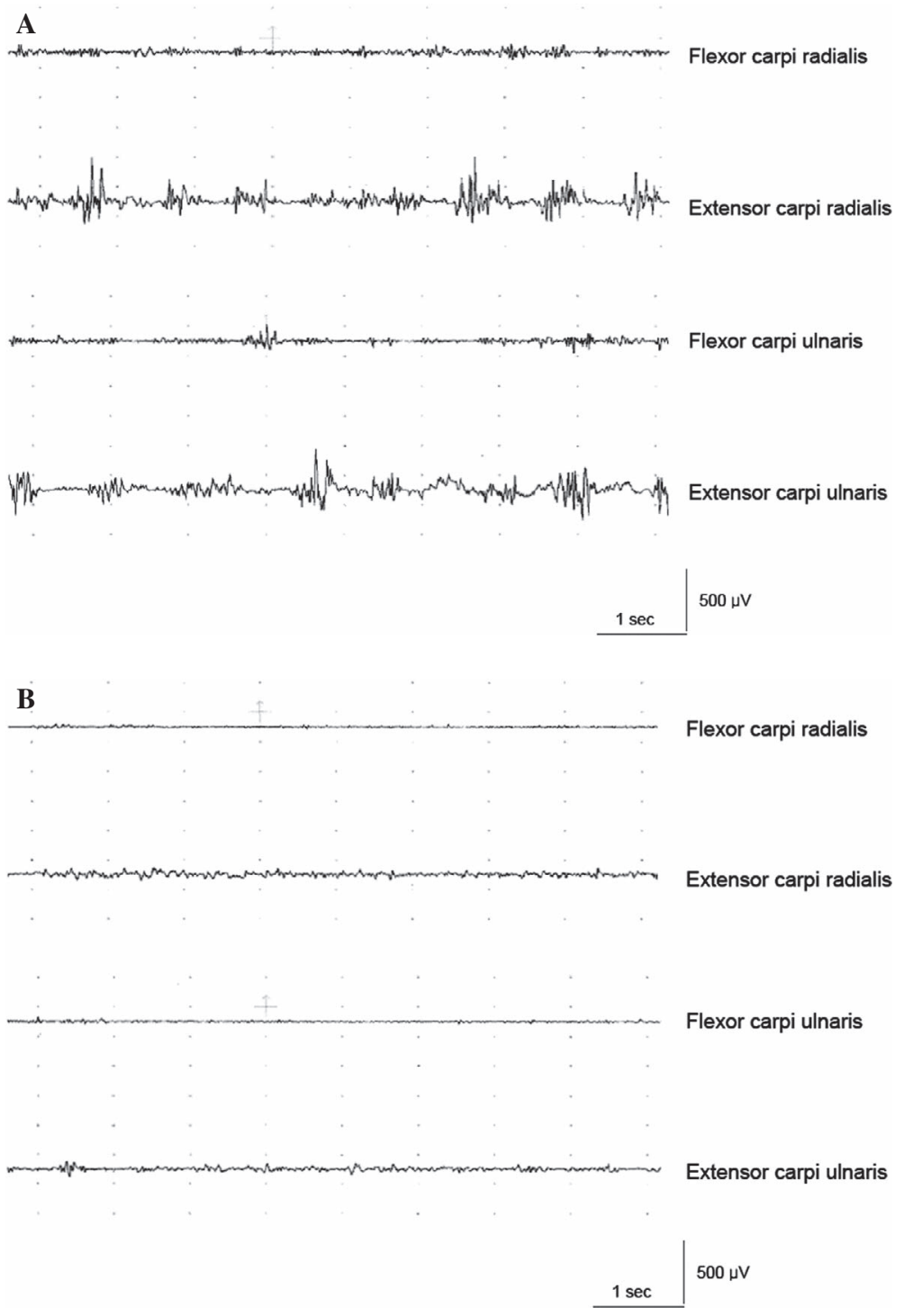

Figure 2. Unrectified surface electromyography (EMG) activities before (A) and after (B) drinking alcohol recorded from the flexor and the extensor muscles of the forearm during handwriting grip posture. Excessive EMG activities were more frequent in the extensor carpi radialis and ulnaris than in the forearm flexor muscles (A). The same procedure was performed 30 minutes after drinking alcohol, and there was nearly complete remission of EMG activities both in the forearm flexor and the extensor muscles (B).

While writing with the left hand (non-dominant hand), she also had a subjective sensation of a spasm in the right hand. This movement was relieved 10 minutes after drinking two cups of beer (approximately $200 \mathrm{~mL}$ ) (Fig. 1B). The effect persisted for about 3 hours.

To identify the physiologic effects of alcohol, a surface electromyography (EMG) was recorded from the flexor and the extensor muscles of the forearm during handwriting grip posture. Excessive EMG activities were more frequent in the extensor carpi radialis and ulnaris than in the forearm flexor muscles during adopting the writing position of the hand (Fig. 2A). The same procedure was performed 30 minutes after drinking alcohol, and there was nearly complete remission of EMG activities both in the forearm flexor and the extensor muscles (Fig. 2B).

The evaluation, including a magnetic resonance imaging of the brain, an electroencephalogram, serum chemistries, a complete blood count, serum ceruloplasmin, thyroid function tests, and genetic testing for the DYT1 mutation in the torsion A gene and $\varepsilon$-sarcoglycan gene (SGCE), were all normal. The dental and ophthalmologic examinations were also normal.

\section{Discussion}

Several hyperkinetic movement disorders share the unusual feature of improving with ingestion of alcohol. The essential tremor or dystonia in some patients can be alleviated with alcohol consumption $(2,3)$. In addition, dramatic responses to ethanol are a defining characteristic of myoclonus-dystonia (4-6).

The present patient had a feature characteristic of primary 
writer's cramp. The unexpected finding was the response of this symptom to alcohol. The mechanism underlying this phenomenon remains unclear; however, several explanations exist. The most plausible explanation is that alcohol may act via brainstem structures, such as the cerebellum and inferior olive, and enhance GABAergic transmission onto dopamine neurons, as in animals $(7,8)$; this view is supported by studies that the reduction of brain GABA may play a role in the clinical symptomatology of writer's cramp (9). Furthermore, ethanol may directly elevate the dopamine level, and therefore it appears to modulate abused behavioral animal and human models (10). In addition to the direct effects of alcohol on the basal ganglia and associated structures, we cannot rule out the possibility that the anxiolytic influence or placebo effect of alcohol may contribute to its beneficial effects on the disease. There have been several reports on the anxiolytic effects of alcohol consumption $(11,12)$. Alcohol can induce the $\mathrm{GABA}_{\mathrm{A}}$ receptor plasticity. $\mathrm{GABA}_{\mathrm{A}}$ receptors modulate anxiety and response to stress; thereafter alcohol possibly lead to decrease level of anxiety and may promote clinical improvement of the disease $(11,12)$.

Similar responses to alcohol have also been described in primary writing tremors (13). Recent neurophysiologic investigations in patients with primary writer's cramp have pointed to the co-existence of features of tremors and dystonia (13). In addition, there exist many controversies as to whether a primary writing tremor is a variant of an essential tremor or a variant of writer's cramp (14). Furthermore, the co-contraction pattern of agonist and antagonist muscle cannot always classify a type of tremor and differentiate tremor from dystonia because variable patterns of contraction were observed in the long-term EMG recording in patients with essential tremor (15). In this regard, the response to alcohol in the present patient may suggest the possibility that this patient may have suffered from a co-existing writing tremor, although the EMG findings of this patient did not correspond to a primary writing tremor.

Another explanation might be associated with the phenotype of the myoclonus-dystonia syndrome, which may have unusual clinical spectra, including writer's cramp or focal dystonia (16). Mutations in the gene, SGCE, are known to be responsible for approximately only one-third of cases (17). In addition, direct genomic DNA sequencing fails to detect SGCE mutations in up to $30 \%$ of familial myoclonus-dystonia cases (18). Therefore, it should be ruled out that the writer's cramp in the present patient might be one of the manifestations of myoclonus-dystonia, although the patient's symptoms were apparently sporadic and the results of genetic analysis for SGCE were negative.

This case represents one further example of the associa- tion between alcohol and movement disorders. Although the exact mechanism is unknown, alcohol might reverse the pathophysiologic changes in the entire basal ganglia circuit in patients with writer's cramp.

The authors state that they have no Conflict of Interest (COI).

\section{References}

1. Rivest J, Lees AJ, Marsden CD. Writer's cramp: treatment with botulinum toxin injections. Mov Disord 6: 55-59, 1991.

2. Jankovic J. Essential tremor: clinical characteristics. Neurology 54: S21-S25, 2000.

3. Gudin M, Vaamonde J, Rodriguez M, Obeso JA. Alcohol sensitive dystonia. Mov Disord 8: 122-123, 1993.

4. Quinn NP. Essential myoclonus and myoclonic dystonia. Mov Disord 11: 119-124, 1996.

5. Kyllerman M, Forsgren L, Sanner G, Holmgren G, Wahlström J, Drugge U. Alcohol-responsive myoclonic dystonia in a large family: dominant inheritance and phenotypic variation. Mov Disord 5: 270-279, 1990.

6. Kinugawa K, Vidailhet M, Clot F, Apartis E, Grabli D, Roze E. Myoclonus-dystonia: an update. Mov Disord 24: 479-489, 2009.

7. Klebe S, Stolze H, Grensing K, Volkmann J, Wenzelburger R, Deuschl G. Influence of alcohol on gait in patients with essential tremor. Neurology 65: 96-101, 2005.

8. Theile JW, Morikawa H, Gonzales RA, Morrisett RA. Ethanol enhances GABAergic transmission onto dopamine neurons in the ventral tegmental area of the rat. Alcohol Clin Exp Res 32: 10401048, 2008.

9. Levy LM, Hallett M. Impaired brain GABA in focal dystonia. Ann Neurol 51: 93-101, 2002.

10. Löf E, Ericson M, Stomberg R, Söderpalm B. Characterization of ethanol-induced dopamine elevation in the rat nucleus accumbens. Eur J Pharmacol 555: 148-155, 2007.

11. Grobin AC, Matthews DB, Devaud LL, Morrow AL. The role of $\operatorname{GABA}(\mathrm{A})$ receptors in the acute and chronic effects of ethanol. Psychopharmacology (Berl) 139: 2-19, 1998.

12. Enoch MA. The role of $\operatorname{GABA}(\mathrm{A})$ receptors in the development of alcoholism. Pharmacol Biochem Behav 90: 95-104, 2008.

13. Bain PG, Findley LJ, Britton TC, et al. Primary writing tremor. Brain 118: 1461-1472, 1995.

14. Byrnes ML, Mastaglia FL, Walters SE, Archer SA, Thickbroom GW. Primary writing tremor: motor cortex reorganisation and disinhibition. J Clin Neurosci 12: 102-104, 2005.

15. Boose A, Spiker S, Jentgens C, Dichagans J. Wrist tremor; Investigation of agonist-antagonist interaction by means of long-term EMG recording and cross-spectral analysis. Electroenceph clin Neurophysiol 101: 355-363, 1996.

16. Chen XP, Zhang YW, Zhang SS, et al. A novel mutation of the epsilon-sarcoglycan gene in a Chinese family with myoclonusdystonia syndrome. Mov Disord 23: 1472-1475, 2008.

17. Schüle B, Kock N, Svetel M, et al. Genetic heterogeneity in ten families with myoclonus-dystonia. J Neurol Neurosurg Psychiatry 75: 1181-1185, 2004.

18. Valente EM, Edwards MJ, Mir P, et al. The epsilon-sarcoglycan gene in myoclonic syndromes. Neurology 64: 737-739, 2005.

(C) 2012 The Japanese Society of Internal Medicine http://www.naika.or.jp/imindex.html 